\title{
PRACTICAL CONSIDERATIONS IN IMPLEMENTING SHIPBOARD POWER SYSTEM RECONFIGURATION CONTROL SCHEMES
}

By:

M. Shasteen

K. Davey

R. Longoria

W. Shutt

IEEE Electric Ship Technologies Symposium (ESTS 2007), Arlington, Virginia, U.S.A., May 21-23, 2007.

PN 318

Center for Electromechanics The University of Texas at Austin PRC, Mail Code R7000

Austin, TX 78712

(512) 471-4496 


\title{
Practical Considerations in Implementing Shipboard Power System Reconfiguration Control Schemes
}

\author{
Michael Shasteen, Member, IEEE, Kent Davey Fellow, IEEE, Raul Longoria*, Member, IEEE, \\ William Shutt
}

\begin{abstract}
The goal of an automatically reconfigurable shipboard power system is to prevent self-inflicted damage and to generate and utilize power in an efficient and reliable manner. To achieve this objective, the system must balance response time with decision accuracy. Speed of calculation is achieved by (1) utilizing a more efficient method of calculating optimization parameters and (2) monitoring indicating parameters to decide when to calculate a new system configuration. In addition to these points, recommendations are made regarding methodologies that account for the influence of the gas turbine power plants expected to form the foundation of future electric shipboard power systems.
\end{abstract}

\section{INTRODUCTION}

A MONG the more significant advantages of an all electric ship are more efficient propulsion drives and significant weapons power. These advantages are enhanced significantly when the shipboard power system (SPS) is designed with power electronic switches allowing grid reconfiguration. A system of coordinated switches will allow the grid to customize the routing of power under variable load.

The synchronization of these switches with the SPS requires intelligent control. This study presents a practical top-down approach to the reconfiguration control challenge. While mitigation of damage in emergency situations is critical, a sensibly-designed system should possess the flexibility to adapt to various mission profiles. A reconfigurable SPS offers the possibility to improve both energy efficiency and fight through capability.

A modified version of the simulation model developed in [4] and [6] is utilized extensively in this investigation. The reconfiguration involves the following four tasks:

1. Simulate the impact of fluctuations in load impedances and switch settings using a dynamic model of the SPS, including 2 generators, 5 loads, and 10 switches.

Manuscript received March 6, 2007. This work was supported in part by the Office of Naval Research.

M. Shasteen is a student at the University of Texas, Austin, TX USA.

K. Davey is with the University of Texas, Center for Electromechanics, 1 University Station R7000, Austin, TX 78712 USA.

R. Longoria is with the University of Texas, Department of Mechanical Engineering, 1 University Station C2200, Austin, TX 78712 USA

W. Shutt is with the University of Texas, Applied Research Laboratories, 1 University Station F0252, Austin, TX 78712 USA.
2. Take current and voltage readings over time to estimate equivalent impedances for transmission lines and parallel loads.

3. Use these readings and a static SPS model to determine the optimal switch configuration for efficiently utilizing generator power within the system limitations.

4. Avoid disconnecting active loads and wait for zero voltage and current crossings while transitioning the dynamic model to the desired state.

It is important to focus on algorithms that can efficiently navigate the nonlinear landscape of this binary optimization problem. This study compares the accuracy and performance of two such algorithms when applied to systems of various sizes. In addition, methods of monitoring system characteristics and potential reconfiguration triggers are presented. The paper also investigates the impact of gas turbine efficiency and specific fuel consumption on the existing SPS model, a critical element in moving toward the practical implementation of automatically controlled reconfiguration of SPS.

\section{OPTIMIZATION PeRformanCE}

Earlier studies of a reconfigurable SPS [6] utilized an exhaustive search approach to determine an optimal configuration, an approach that guarantees discovery of the best possible solution. The algorithm operates identically in each iteration cycle; and the computation time should not fluctuate significantly. The exhaustive search proved valuable in illustrating the effectiveness of the overall reconfiguration approach when applied to a 10-switch system, as shown in Fig. 1; however, as the number of switches in the system increases, the number of possible solutions increases exponentially. For a system with $n$ switches, the number of options is $2^{n}$. This means there are 1024 configurations for a 10-switch system, 65,536 configurations for a 16-switch system, and 16.8 million configurations for a 24-switch system. Clearly, an efficient optimization routine must be utilized when analyzing such large yet practically significant systems. 


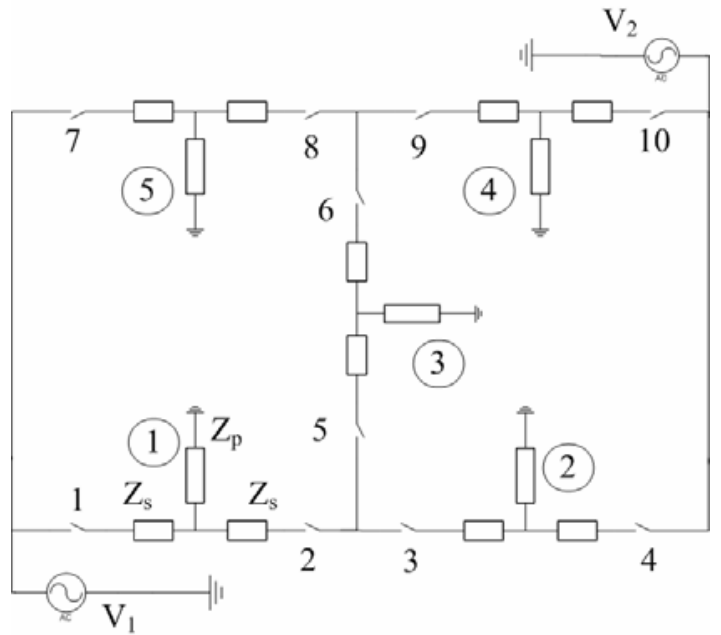

Fig. 1 5-Load, 10-switch system used for simulation.

\section{A. Pattern Search}

One type of direct search algorithm, the pattern search [1] and [2], was investigated and its performance compared to that of the exhaustive search. The pattern search does not require a differentiable or continuous objective function. It is tailored for continuous optimization problems but can be made applicable to discrete problems by appropriate use of penalty functions. The algorithm evaluates the objective function at various coordinates in the solution space surrounding an initial point. Using the Positive Basis $2 \mathrm{~N}$ polling method, these $2 \mathrm{n}$ points are found by adding and subtracting the mesh size to each of the dimensions of the initial point vector. The process is then repeated with a different initial point and mesh size, depending on whether a smaller value of the objective function is found (a successful poll). Parameters like the mesh contraction and mesh expansion factors can be modified to accelerate the search procedure and to some extent prevent the algorithm from settling in local minima.

Three issues in particular make the reconfigurable SPS a challenge for the pattern search algorithm:

1. Switches must either be open or closed, making this a discrete (binary) optimization problem

2. If possible, the optimal solution should not violate any constraints.

3. Neither the above two factors nor should the original power term dominate the search.

All three conditions may be satisfied by using the following objective function:

$\mathfrak{I}=\frac{\left(\sum I_{s}{ }^{2} R_{s}-\sum I_{p}{ }^{2} R_{p}\right)_{n}}{\left(\sum I_{s}{ }^{2} R_{s}-\sum I_{p}{ }^{2} R_{p}\right)_{n-1}}+\sum(x-0.001)(0.999-x)+C$

where

$C=\sum I_{p}<I_{p_{-} \min }+\sum I_{s}>I_{s_{-} \text {rated }}+\sum P_{\text {gen }}>P_{\text {rated }}$

The second term, a convex penalty function, encourages binary solutions for all switches [7]. The constraint penalty function (C) penalizes (adds one to) the objective function for each constraint violated. Constraints include minimum load current, maximum line current, and generator power capacity. The numerator of the first term is identical to the complete objective function used for the exhaustive search. However, to ensure all three terms are of similar magnitude, this value (n) must be normalized against the same quantity evaluated at the present switch configuration (n-1).

\section{B. Latin Hypercube Sampling}

Despite the normalization of the objective function, pattern search iterations sometimes converge on a local minimum or solution where constraints are violated. The occurrence of these undesired solutions appears to be highly dependent on the starting point selected for the algorithm. Running the pattern search multiple times and using a Latin Hypercube Sampling (LHS) technique to create a distribution of starting points aids the pattern search in overcoming these obstacles. LHS ensures that the starting points chosen are well-distributed throughout the solution space, increasing the likelihood of finding a valid global minimum [7].

\section{Performance and System Size}

In addition to the 10-switch system developed in earlier efforts, two additional systems were modeled to measure the performance of the pattern search against the exhaustive search for varying system size. Fig. 2 below illustrates the designs of each SPS.
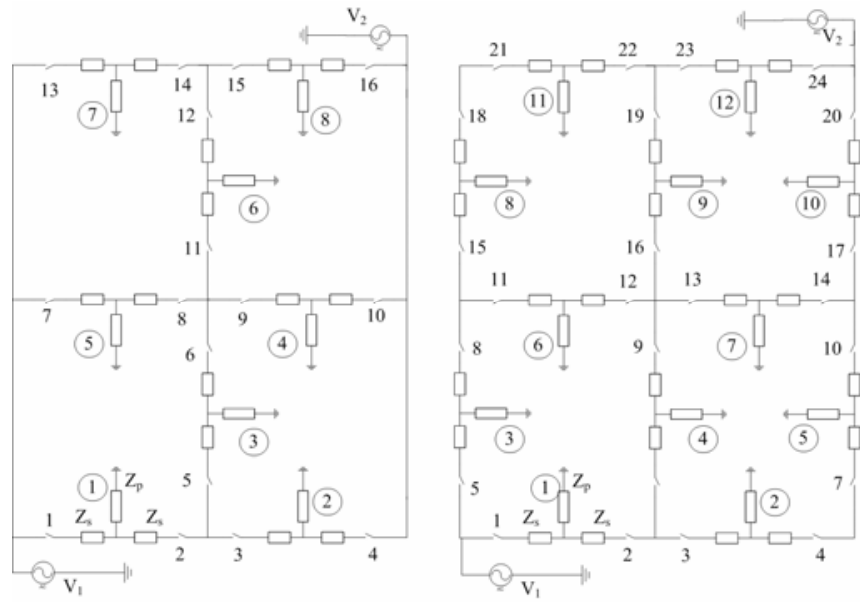

Fig. 2 8-Load, 16-switch system (left) and 12-load, 24-switch system (right), used for simulation.

Both search methods were tested repeatedly with all three systems on a $2.8 \mathrm{GHz}$ Pentium IV computer. The results of these tests, summarized in Fig. 3 and Table 1, confirm the expected outcomes. For a 10 -switch system, the average exhaustive search time ( 0.34 seconds) was actually less than that of the pattern search (0.46). As the system size increases, the superior performance of the pattern search approach is revealed. In fact, the exhaustive search was not used on the 24-switch system due to computational time (greater than 10 hours on a $3 \mathrm{GHz}$ Pentium IV) [3]. The results in Table 1 show that the pattern search achieves a 
very high success rate. In fact, even the "non-optimal" solutions returned by the algorithm were not a complete failure. Any solutions that violate a constraint can easily be identified and rejected, while the remaining local minima were actually within $10 \%$ of the optimal values in all systems.

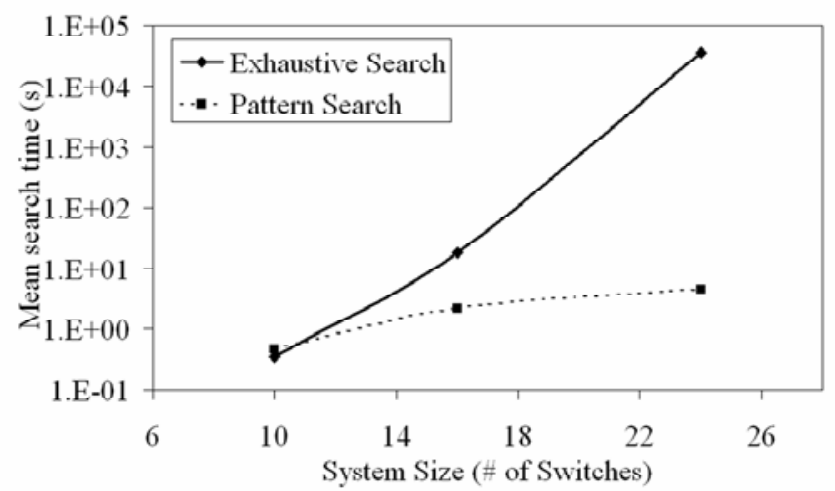

Fig. 3 Speed of pattern search and exhaustive search for various system sizes.

Table 1 Pattern Search Accuracy

\begin{tabular}{|l|c|c|c|}
\hline \# Switches & 10 & 16 & 24 \\
\hline Optimal solutions $^{1}$ & $99 \%$ & $93 \%$ & $94 \%$ \\
\hline Constraints violated & $1 \%$ & $5 \%$ & $0 \%$ \\
\hline Local minima & $0 \%$ & $2 \%$ & $6 \%$ \\
\hline
\end{tabular}

${ }^{1}$ Any solution with an objective function value within $1 \%$ of the determined global minimum is considered optimal.

\section{SYSTEM MONITORING}

Just as the search method is important to the success of a reconfigurable SPS, so are the criteria of determining when reconfiguration may be necessary. Continuous optimization initially proved useful in revealing typical trends in [6]. In an operational system this practice would be both impractical and unnecessary. The optimal system configuration determined at a given time and state should logically remain the best solution until the system state changes significantly. This criterion requires formulation of a standard by which to judge whether a change in state is significant.

\section{A. Change of Load Impedances}

During routine operation, all parameters of the notional SPS evaluated in this study are assumed time invariant save two - switch configuration and load impedances. From this observation, it can be concluded that significant changes in SPS states would require variation in load impedances, assuming switch configuration is held constant. Thus by monitoring loads for impedance changes, the system controller could determine whether reconfiguration is necessary.

One way to reduce the computational requirements is to monitor each load's impedance for a percent change in value. This idea was tested with the aforementioned 5-load, 10-switch system. First, an initial optimal switch configuration was determined using the nominal values for each of the load impedances. Then, a single load was reduced by $5 \%$, and the optimization routine run again. This process was repeated for this same load until the algorithm indicated a change in the preferred switch configuration. Repeating this procedure for each of the five loads in the system yielded the results graphed in Fig. 4. A definite correlation appears between the impedance of a load and the percent change required to initiate reconfiguration. This trend appears to be highly dependent on system parameters and does not provide sufficient evidence to determine the nature of this relationship.

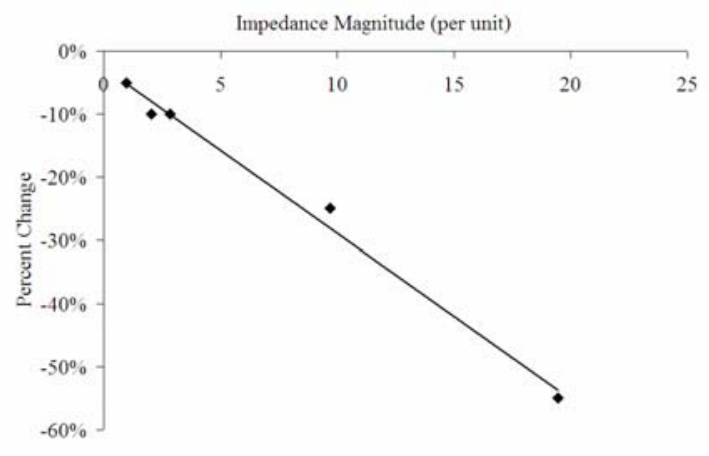

Fig. 4 Minimum percent impedance change required for reconfiguration in each of the 5 loads.

\section{B. System Equivalent Impedance}

Because transmission line impedances are usually orders of magnitude smaller than load impedances, the 5-load SPS in Fig. 1 could be represented as a group of 5 parallel loads, as shown in Fig. 5. Using this adapted model, the effective impedance of the network of parallel loads is calculated. This system equivalent value can then be monitored to detect significant load changes. Reviewing the data generated for Fig. 4. above demonstrates the merit of this concept. While slowly decreasing any of the five loads, the optimal switch configuration remains unchanged until the system equivalent impedance falls $2-3 \%$ from the initial value. Table 2 offers a numerical visualization of this trend.

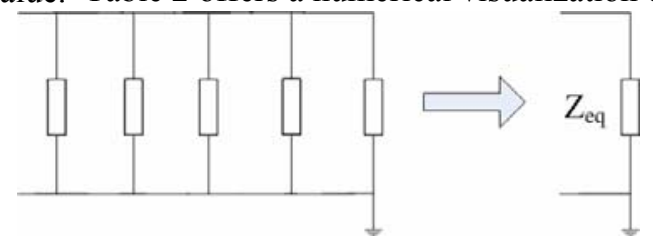

Fig. 5 Parallel load representation of the SPS

Table 2 System Equivalent Impedance Change Due to Individual Load Change 


\begin{tabular}{|c|c|c|c|c|c|}
\hline $\begin{array}{c}Z_{\text {load }} \\
\text { Change }\end{array}$ & $\begin{array}{c}\text { Load } \\
1\end{array}$ & $\begin{array}{c}\text { Load } \\
2\end{array}$ & $\begin{array}{c}\text { Load } \\
3\end{array}$ & $\begin{array}{c}\text { Load } \\
4\end{array}$ & $\begin{array}{c}\text { Load } \\
5\end{array}$ \\
\hline$-5 \%$ & $-1 \%$ & $0 \%$ & $0 \%$ & $-1 \%$ & $-3 \%$ \\
\hline$-10 \%$ & $-3 \%$ & $0 \%$ & $-1 \%$ & $-2 \%$ & $-5 \%$ \\
\hline$-15 \%$ & $-4 \%$ & $0 \%$ & $-1 \%$ & $-3 \%$ & $-8 \%$ \\
\hline$-20 \%$ & $-6 \%$ & $-1 \%$ & $-1 \%$ & $-4 \%$ & $-11 \%$ \\
\hline$-25 \%$ & $-7 \%$ & $-1 \%$ & $-2 \%$ & $-5 \%$ & $-15 \%$ \\
\hline$-30 \%$ & $-9 \%$ & $-1 \%$ & $-2 \%$ & $-7 \%$ & $-18 \%$ \\
\hline$-35 \%$ & $-12 \%$ & $-1 \%$ & $-3 \%$ & $-8 \%$ & $-22 \%$ \\
\hline$-40 \%$ & $-14 \%$ & $-2 \%$ & $-3 \%$ & $-10 \%$ & $-26 \%$ \\
\hline$-45 \%$ & $-17 \%$ & $-2 \%$ & $-4 \%$ & $-12 \%$ & $-30 \%$ \\
\hline$-50 \%$ & $-19 \%$ & $-2 \%$ & $-5 \%$ & $-14 \%$ & $-34 \%$ \\
\hline$-55 \%$ & $-23 \%$ & $-3 \%$ & $-6 \%$ & $-17 \%$ & $-39 \%$ \\
\hline$-60 \%$ & $-27 \%$ & $-4 \%$ & $-7 \%$ & $-20 \%$ & $-44^{\circ} \%$ \\
\hline
\end{tabular}

Highlighted cells indicate an optimal switch configuration different from the nominal value.

\section{Generator IMPACT ON Optimization}

Previous work on the reconfiguration of the SPS has focused on maximizing power delivered to the loads while minimizing transmission line losses. This method facilitates investigation of system dynamics of the SPS; however, the fundamental goal should be to optimize the power at its source. Gas turbines currently power the SPS generators aboard all new naval vessels. By minimizing the total fuel consumed by all turbines on the ship while ensuring that loads receive the necessary power, the SPS will achieve its optimal switch configuration.

The efficiency of all turbines increases as power output approaches peak rated levels; this fact is explored more thoroughly in [4]. Thus, it can be concluded that even though the SPS of a ship may allow multiple turbines to provide power, the system operates more economically with a single turbine running close to rated capacity. This concept contrasts with the goal of maximizing power delivery less transmission loss, where the switch configuration is driven primarily by the need to control power flow. Transmission loss optimization is highly dependent on turbine generator location. Fuel efficiency optimization depends on turbine power output and only secondarily on location in the grid.

\section{A. Modified Objective Function}

Davey [5] proposed an empirically-derived representation for a turbine specific fuel consumption and power curve in the form,

$$
\xi=\xi_{0}+\frac{\left(\xi_{2}-\xi_{0}\right)}{1-e^{-m}} \cdot\left(1-e^{-m\left(\frac{P-P_{\min }}{P_{\max }-P_{\min }}\right)}\right)
$$

where $\xi_{0}$ and $\xi_{2}$ represent the specific fuel consumption (in $\mathrm{kg} /(\mathrm{kW}-\mathrm{Hr})$ ) at the lowest and rated power settings, respectively, and $\mathrm{m}$ is a turbine-specific exponent. This relationship was integrated into the objective function as follows:

$\mathfrak{J}=\frac{\left(\sum P_{\text {gen }} \cdot \xi_{\text {gen }}\right)_{n}}{\left(\sum P_{\text {gen }} \cdot \xi_{\text {gen }}\right)_{n-1}}+\sum(x-0.001)(0.999-x)+C$
The first term has been replaced by the normalized sum of the instantaneous fuel consumption rates of all turbines.

\section{B. Model Results}

This new objective function was tested on the 5-load, 10switch system explored earlier in the paper. Load impedances were selected such that the peak initial system power demand totaled to 0.8 per unit, just below the capacity of a single generator. Using a step input on the load factor for the central load 3, this power demand was quickly increased to 1.2 per unit. Optimization was performed in this scenario using both objective functions (transmission loss and fuel efficiency). The results for transmission loss optimization are illustrated in Fig. 6. At $80 \%$ of peak power demand, both turbines operate at power ratings far below peak capacity. Although the generators were modeled identically, there exists a difference in optimal power output that can be attributed to an imbalance in the impedances specified for loads and transmission lines. Therefore, generator location plays a role in determining power production of each turbine.
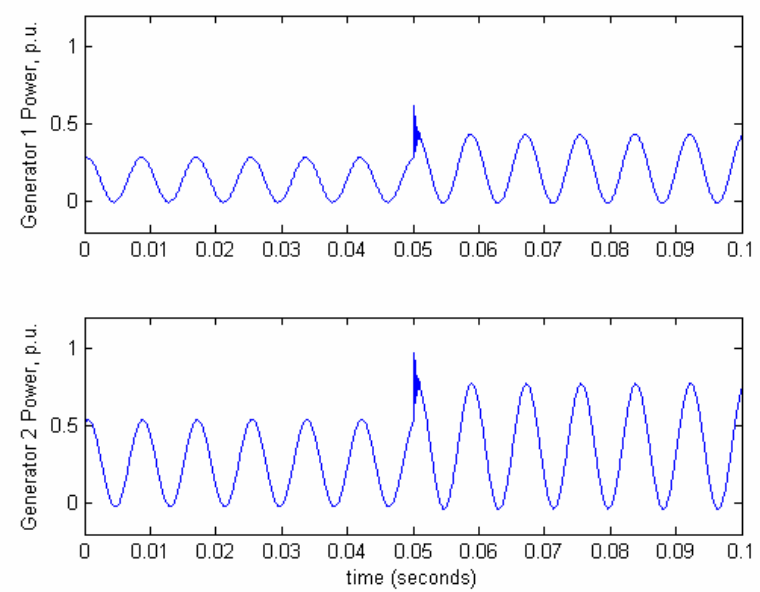

Fig. 6 Instantaneous single-phase turbine generator power output for transmission loss optimization.

When the switch setting is optimized based on fuel efficiency as in Eq. 3, the outcome is illustrated in Fig. 7. Note that at $80 \%$ total power demand, switches 4 and 10 (shown in Fig. 1) open, leaving turbine number 1 as the sole power generator for the entire SPS. If the demand changes to $120 \%$, switching transients have decayed, and the proper zero crossings have been satisfied, the switches close, reconnecting turbine 2 to the grid. The most efficient point on the combined specific fuel consumption curve lies where both turbines produce approximately the same amount of power. 

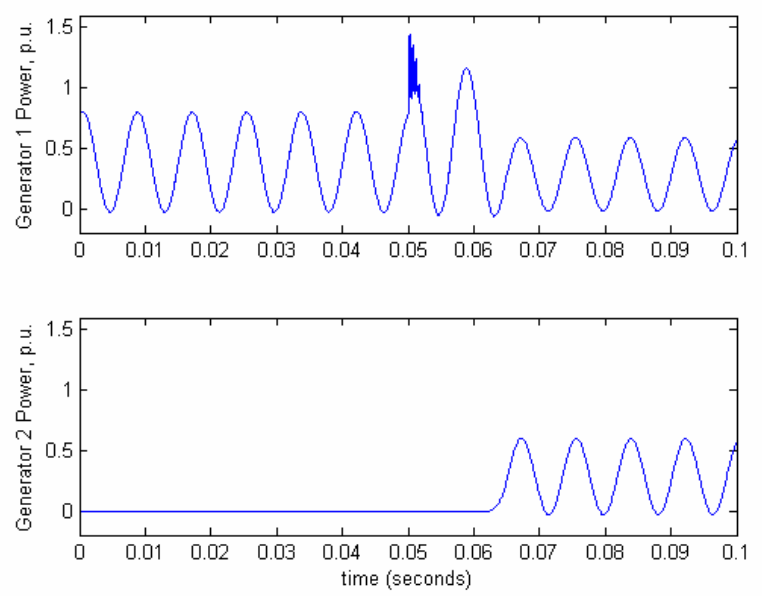

Fig. 7 Instantaneous single-phase turbine generator power output for transmission loss optimization.

\section{CONCLUSIONS}

An approach to control reconfiguration has been illustrated that optimizes the amount of fuel consumed by the system. Certain aspects of the approach were demonstrated on a simulation model to verify their effectiveness. When combined with Latin Hypercube Sampling, the pattern search provides accurate and timely solutions to the optimization problem, even when the system size and complexity increases drastically. The concept of system equivalent impedance appears to be a promising method for monitoring the SPS for significant changes and triggering reconfiguration; significant impedance change magnitudes will no doubt be system dependent. Including turbine generator specific fuel consumption with the optimization process produces a system designed to capitalize on efficiency. Turbine grid tie-in points become secondary to turbine specific fuel consumption.

\section{REFERENCES}

[1] R. M. Lewis and V. Torczon, "Pattern Search Methods for Linearly Constrained Minimization," SIAM Journal on Optimization, Vol. 10, Number 3, 2000, pp. 917-941.

[2] C. Audet and J. E. Dennis Jr., "Analysis of Generalized Pattern Searches," SIAM Journal on Optimization, Vol. 13, Number 3, 2003, pp. 889-903.

[3] K. Davey and Robert Hebner, "Reconfiguration: A Tool for Designing New Ships," 2005 IEEE Electric Ship Technologies Symposium Proceedings, Philadelphia, Pennsylvania, July 25-27, 2005, pp. 81-85.

[4] K. Davey, R. Longoria, W. Shutt, J. Carroll, K. Nagaraj, J. Park, T. Rosenwinkel, W. Wu, and A. Arapostathis, "Reconfiguration in Shipboard Power Systems," American Control Conference (ACC '07), New York, NY, July 11-13, 2007. Accepted for publication/presentation.

[5] K. Davey, "Ship component in hull optimization," Marine Technology Society Journal, Vol. 39, No. 2, pp. 35-42, 2005.

[6] Jerad Park, "Dynamic modeling and analysis of an automatic reconfigurable shipboard power system," MS Thesis, University of Texas at Austin, 2006.

[7] Kent Davey, "Latin Hypercube Sampling and Optimization in an Electric Ship," Electric Ship Research and Development Consortium August Meeting, Lafayette, Indiana, August 17-18, 2006. 